\title{
Sequence Verification of Oligonucleotides Containing Multiple Arylamine Modifications by Enzymatic Digestion and Liquid Chromatography Mass Spectrometry (LC/MS)
}

\author{
Lan Gao, ${ }^{\text {a Li Zhang, }}$, Bongsup P. Cho, ${ }^{\text {b }}$ and M. Paul Chiarelli ${ }^{\mathrm{a}}$ \\ ${ }^{a}$ Department of Chemistry, Loyola University, Chicago, Illinois, USA \\ b Department of Biomedical and Pharmaceutical Sciences, College of Pharmacy, University of Rhode Island, \\ Kingston, Rhode Island, USA
}

An@nalytical@ethod@or@he@tructure@ifferentiation@f@rylamine@nodified@ligonucleotides (ODNs)@sing@n-line@C/MS@nalysis@f@aw@xonuclease@igests@s@escribed.(\$ix@ifferent dodeca@DDNs@erived@from@the@reaction@of $\mathrm{CN}$-acetoxy- $\mathrm{N}$-(trifluoroacetyl)-2-aminofluorene with@he@odeca@ligonucleotide(5'-CTCGGCGCCATC-3'@re@solated@nd@equenced@ith $₫$ his

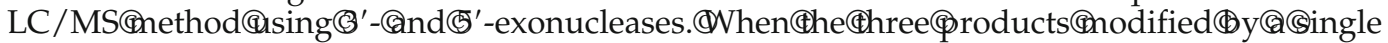

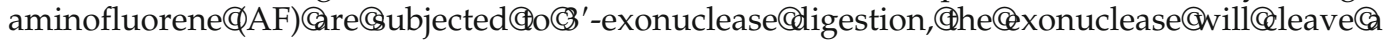
modified@ucleotide@ut@when@i-AF@nodified@DNs@re@nalyzed@he(3'-exonuclease@eases

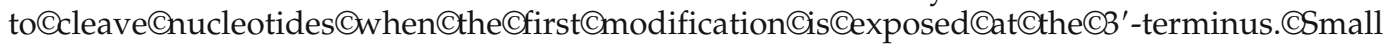
abundances@f@DN@ragments@ormed@y@he@leavage@f@n@AF-modified@ucleotide@vere observed@when@wo@f@he@hree@i-AF@modified@DNs@ere@ubjected@o@্s'-exonuclease

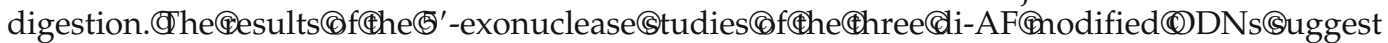
that@s@he@umber@f@unmodified@bases@etween@wo@modifications@n@n@DN@equence increases, (he@asier $₫$ (†) The@esults@@is@tudy@ndicate@hat $₫$ he@L/MS@nethod@escribed@here@vould@e@seful@n sequencing@DNs@nodified@y@ultiple@rylamines@o@e@used@s@emplates@or@ite-specific

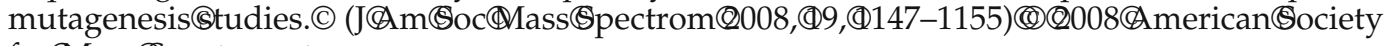
for@Mass $\$$

I nterest@n@he@nalysis@f@arcinogen-modified@u-

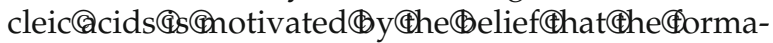
tion@f@hese@ompounds@nay@ead@o@he@evelopment@f@umors.@he@bility@f@@articular@nodification

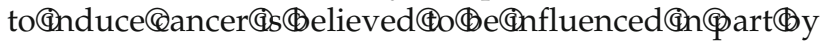

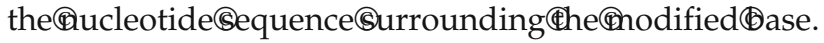
In@articular, (he@dentities@f@he@ases@urrounding theథesion@ite@re@trongly@uggested@o@nfluence@he rate@of@mutation@and@DNA@repair@1,C2].CThe@most rigorous@way@to@arry@out@ite-specific@mutagenesis studies@ould(థeథo@nsert@@hort(1)NA@trand@ontaining@well-characterized@modifications@at@specific@sites into@\$articular@ector@nd@hen@etermine@he@epair and@mutation@utcomes $₫ 1, \mathbb{C}, \mathbb{C}$ ].CThe@uccess@f@hese studies@s@redicated@n@he@reparation@nd@haracterization@f@arcinogen-modified@ligodeoxyribonucleotides@ODNs)@with@known@equences.CArylamines, @uch as@2-aminofluorene,@vill@ind@o@he@8@osition@f@ guanine@base@hrough@@itrenium@on@5]@o@orm@AF adduct.đhe@umber@f@equential@somers@btained@n the@eaction@f@n@ctivated@rylamine@ith@n@DN@ill

Address reprint requests to Dr. M. P. Chiarelli, Department of Chemistry, Loyola University, 1068 W Sheridan Rd., Chicago, IL 60626, USA. E-mail: mchiare@luc.edu depend@nథhe@umber@f@uanine@ases@resent@in@he sequence.

SequenceCverification@using@tandem@MSCmethods have@been@used@with@varying@degrees@of@success@in obtaining@tructural@nformation@rom@modified@ligonucleotides $\Phi 6-8] . @$ applications@f( $₫$ andem@MS@nethods for@he@tudy@f@arcinogen-modified@DNs@have@een limited@ue@o@he@ccurrence@f@onspecific@ragmentation@hat@nay@nake@equence@ssignments@ery@ifficult.@o@ate,@nost@fforts@o@equence@amaged,@hort oligomers@25@ucleotides@r(less)@have@nvolved@xonuclease@igestion@ollowed@y@@ample@urification@tep combined@ith@natrix-assisted 4 ser@esorption@onization (MALDI)@ombined@vith@ime-of-flight@TOF)@nass@pectrometry@9@13].đnCthese@studies@B' - or 5'-exonuclease digests@re@ampled@t@ifferent@imeGintervals@and spotted@on@a@laser@probe@for@mass@analysis.CThe MALDI mass spectra show a series of ODNs whose mass differences correspond to the nucleotides cleaved from the terminus. This strategy is often referred to as ladder sequencing.

Previous ESI-MS studies of ODN digestion products [14-18] have been based on off-line preparation and desalting followed by mass analysis via direct infusion. Direct on-line HPLC-ESI-MS for structure identification 
of DNA adducts using exonuclease digestion has not been accomplished to date. In all cases, adduct ions formed from salts used in the digestion process have limited the amount of structural information that may be obtained in a mass spectrum. An off-line desalting step $[16,17]$ is often used to clean up the samples before ESI-MS (or MALDI) analysis. Here we demonstrate the first methodology using on-line HPLC sample clean up and separation coupled with ESI-MS for analysis of the enzyme digestion products of DNA adducts. Methods based on LC/MS are attractive because they would permit the direct analyses of ODN digests as a function of time without any sample pretreatment. The LC/MS analyses of a variety of dodeca ODNs containing the NarI sequence, 5'-CTCG $\mathrm{G}_{2} \mathrm{CG}_{3} \mathrm{CCATC}-3^{\prime}$, containing different aminofluorene modifications in different positions are presented. The NarI sequence is a well-known hot spot for deletion mutations in Escherichia coli [19-21]. The mono-AF adducts characterized here have been used for structure studies [22].

\section{Materials and Methods}

\section{Sample Preparation}

An unmodified dodeca ODN, 5'-CTCGGCGCCATC-3', was treated with incremental amounts of $N$-acetoxy- $N$ (trifluoroacetyl)-2-aminofluorene in a $\mathrm{pH} 6.0$ sodium citrate buffer at $37^{\circ} \mathrm{C}$ for $18 \mathrm{~h}$ [4]. Formation of a covalent adduct at the C8-position of guanine was followed by hydrolysis of the trifluoroacetyl group to yield an AF-modified ODN. The reaction mixtures were purified by reverse phase HPLC, which consisted of a Hitachi (Pleasanton, CA) EZChrom Elite system with a L2450 diode array as a detector. The purification system employed a Waters (Milford, MA) XTerra C18 column $(10 \times 50 \mathrm{~mm}, 5 \mu \mathrm{m})$ with a 60-min gradient system involving $3 \%$ to $15 \%$ acetonitrile in $\mathrm{pH} 7.0$ ammonium acetate buffer $(0.10 \mathrm{M})$ with a flow rate of $2.0 \mathrm{~mL} / \mathrm{min}$. Each product was purified by repeated HPLC injections. The chromatograms associated with the product isolation and purification are shown in Figure 1. Six different AF-modified products were isolated and throughout the following discussion they are referred to as products (P1-P6) based about their elution (Table 1). Throughout the discussion that follows, the position of the guanines within the chain will be referred to as G1, G2, and G3 going from the 5'- to the 3'-end, consistent with Figure 2.

\section{Enzymatic Digestion}

Snake venom phosphodiesterase ( $3^{\prime}$-PDE) and bovine spleen phosphodiesterase (5'-PDE) were acquired from Worthington Biochemical (Lakewood, NJ) and used without purification. The $3^{\prime}$-PDE cleaves terminal nucleotides sequentially from the $3^{\prime}$-end of the ODN, while the 5'-PDE cleaves terminal nucleotides sequentially from the $5^{\prime}$-end of the ODN. Enzymatic digestions were carried out in polypropylene 96-well plates at ambient temperature $\left(24^{\circ} \mathrm{C}\right)$. Each well contained the following reagents: $0.75 \mu \mathrm{L}$ of $1 \mathrm{mg} / \mathrm{mL}$ aqueous solution of each ODN, $3.75 \mu \mathrm{L}$ of $1 \mathrm{unit} / \mathrm{mL}$ snake venom exonuclease aqueous solution, $3.75 \mu \mathrm{L}$ of $20 \mathrm{mM} \mathrm{MgCl} 2$ solution, and $3.75 \mu \mathrm{L}$ of $0.5 \%$ ammonium hydroxide. The final $\mathrm{pH}$ of the reaction mixture was 8.8. After mixing for $30 \mathrm{~s}$, the 96-well plate was loaded onto an autosampler, and the untreated digest samples were injected at $10 \mathrm{~min}$ intervals up to $200 \mathrm{~min}$. The bovine

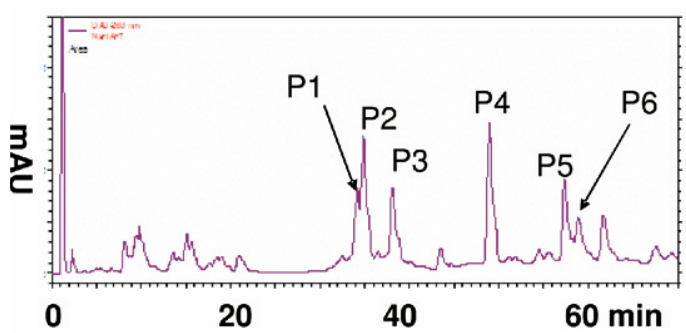

(a)

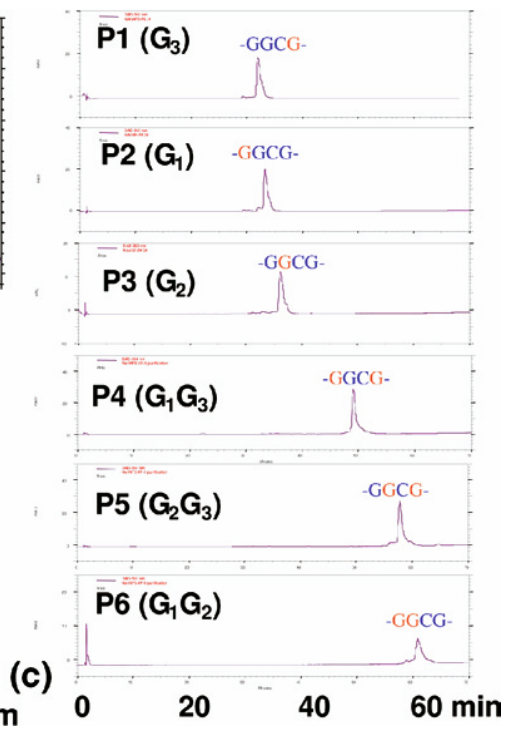

Figure 1. (a) HPLC chromatogram of a mixture from reaction between a 12-mer sequence and $N$-trifuloroacetyl-N-acetoxy-2-aminofluorene. The mono- (G3, G1, G2) and di- (G1G3, G2, G3, G1G2) $\mathrm{AF}$ adducts eluted in the 30-40 and 50-60 min ranges, respectively. (b) On-line UV spectra of the control (unmodified dodecamer), mono- and di-AF adducts. (c) Purity check of all the fractions. 
Table 1. Calculated and measured molecular weights for the AF-modified dodecaODNs used in this study

\begin{tabular}{cccccc}
\hline Sample number & Retention time (minutes) & Sequence $\left(5^{\prime}-3^{\prime}\right)$ & Calculated mass & Measured mass & Error (ppm) \\
\hline \hline P1 & 34.0 & CTCGGCG(AF)CCATC & 3767.69 & 3767.67 & 5.3 \\
P2 & 34.9 & CTCG(AF)GCGCCATC & 3767.69 & 3767.67 & 5.3 \\
P3 & 38.0 & CTCGG(AF)CGCCATC & 3751.69 & 3751.59 & 27 \\
P4 & 49.1 & CTCGG(AF)CG(AF)CCATC & 3727.68 & 3727.63 & 13 \\
P5 & 57.2 & CTCG(AF)G(AF)CGCCATC & 3742.68 & 3742.59 & 24 \\
P6 & 58.9 & CTCG(AF)GCG(AF)CCATC & 3743.68 & 3743.73 \\
\hline
\end{tabular}

spleen exonuclease digests were prepared like the snake venom digests described above. The following components were added to each well: $0.75 \mu \mathrm{L}$ of the ODN aqueous solution at a concentration of $1 \mathrm{mg} / \mathrm{mL}$, $1.6 \mu \mathrm{L}$ of $1 \mathrm{unit} / \mathrm{mL}$ bovine spleen exonuclease solution and $10 \mu \mathrm{L}$ of $10 \mathrm{mM}$ NH4OAc (pH 6.7) buffer. The mixture was allowed to react for various time periods up to $200 \mathrm{~min}$ at ambient temperature before injection.

\section{LC-TOF-MS}

A Shimadzu (Columbia, MD) HPLC system consisting of one SCL-10Avp controller, one SIL-10Advp autosampler, and three LC-10Advp pumps interfaced to a Waters LCT Time-of-Flight mass spectrometer was utilized for LC/MS analysis of the oligonucleotide digests under study. HPLC analyses were carried out at ambient temperature $\left(24^{\circ} \mathrm{C}\right)$ with a Phenomenex (Torrance, CA) Aqua C18 $50 \times 1.0 \mathrm{~mm}$ column (particle size $5 \mu \mathrm{m}$, $125 \AA$ pore size). Solvent A consists of $5 \mathrm{mM}$ ammonium acetate (NH4OAC), $5 \mathrm{mM}$ dimethylbutyl amine (DMBA), and was adjusted to $\mathrm{pH} 5.6$ with acetic acid (about $6.5 \mathrm{mM}$ ). Solvent B contains $0.1 \%$ formic acid in pure acetonitrile. Solvent $C$ was pure acetonitrile. Pumps A and B were running in a binary gradient mode at an initial flow rate of $200 \mu \mathrm{L} / \mathrm{min}$. Pump C delivered acetonitrile at $50 \mu \mathrm{L} / \mathrm{min}$ to increase the ionization efficiency by post-column mixing with LC eluent. A $2.0 \mu \mathrm{m}$ pore size stainless steel frit was placed in front of the column to prevent clogging. A Waters (Milford, MA) LCT time-of-flight (TOF) mass spectrometer was utilized to detect oligodeoxynucleotides introduced into the system from HPLC through an electrospray ionization probe. All TOFMS spectra were acquired in the negative ion mode over a 300 to 2000 $\mathrm{m} / \mathrm{z}$ range at the rate of $1 \mathrm{~s}$ per scan.

\section{Results and Discussion}

\section{Experimental Design}

The goal of this study is to evaluate an analytical method for the characterization of arylamine-modified oligonucleotides that would be used as primers in site-specific mutagenesis studies. The generation of such primers involves reacting a carcinogen with an oligonucleotide of known sequence. The individual reaction products are isolated and mass analyzed to determine the positions of the arylamine modification at different sites in the ODN chain. The molecular masses are measured to determine the number of modifications each ODN reaction product contains and

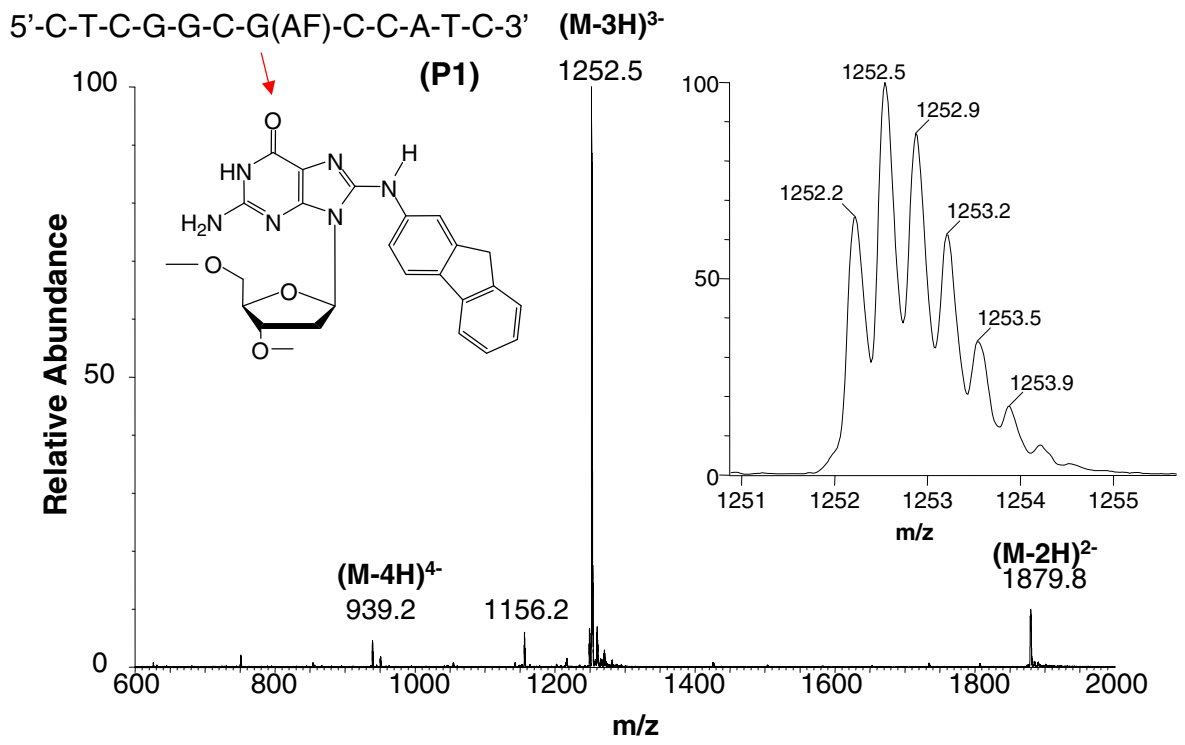

Figure 2. ESI TOF mass spectrum of the ODN product 1 . The structure of AF-adduct $\left[N-\left(2^{\prime}-\right.\right.$ deoxyguanosin-8-yl)-2-aminofluorene in the NarI sequence is shown in the inset. 
then sequenced to determine which bases are modified. Enzymatic digestion coupled with LC/MS is used to differentiate the structures of the different ODN isomers produced in the modification of the dodeca ODN template. Experimental strategies for sequencing modified ODNs using exonuclease digestion and MALDI mass analysis have been presented as though the nucleotide sequence around the modification was unknown. The sequence and position of the modification in an ODN is inferred through the observation of a series of protonated ODN fragments whose $m / z$ values differ by the mass of the terminal nucleotide cleaved by the $3^{\prime}$ - or $5^{\prime}$-exonuclease. The position of the modification is established by comparing mass spectra of $3^{\prime}$ - and 5 '-exonuclease digestions, since modified nucleotides are not observed to be cleaved in these studies. In this particular study, all the modified ODNs have the same backbone sequence, so the key to differentiating positional isomers is to identify ODN digest ions that are specific for a particular sequential isomer. Since arylamines modify only the guanine bases, one may determine the position of a modification through the observation of fragments whose masses differ by the mass of an unmodified guanine nucleotide.

\section{Molecular Weight and UV Absorption Analysis of ODN Reaction Products}

Molecular weight and UV absorption analysis of the individual components isolated from the reaction mixture indicate that six products were formed. A comparison of the UV absorbance spectra in Figure $1 \mathrm{~b}$ of products P1-P3 and products P4-P6 suggest that they possess one and two AF groups, respectively. These observations are consistent with the molecular weight measurements of the all six reaction products summarized in Table 1. All the ODN adducts analyzed here were determined to be guanine C8-substituted adducts by digestion to single 2 -deoxynucleosides and cochromatography with a $N$-[deoxyguanosin-8-yl]-2aminofluorene standard.

Figure 2 is a molecular weight spectrum of $5^{\prime}$ CTCGGCG(AF)CCATC-3' (P1) acquired from the digest mixture immediately after its preparation. The LC/MS spectra of the intact ODNs and digest fragments do not show significant abundances of ions formed by cation exchange with a proton. The most prominent ion in these spectra is the $(\mathrm{M}-3 \mathrm{H})^{3-}$ ion. Smaller abundances of the $(\mathrm{M}-2 \mathrm{H})^{2-}$ and $(\mathrm{M}-4 \mathrm{H})^{4-}$ are observed in these spectra as well. We have found that the molecular weight of the dodeca ODNs may be determined with a mass accuracy of 5-27 parts per million (ppm) with one or two picomoles of ODN injected (Table 1). The mass accuracies achieved in these experiments compare favorably with those obtained in MALDI ladder sequencing studies conducted earlier [9-13]. The molecular weights are calculated from a weighted average of all the sample-specific ions in each isotopic cluster. The precision associated with these measurements is sufficient to assign the composition of the ODN reaction products in this study, given that the minimum mass difference one might expect between ODN fragments is $2 \mathrm{Da}$ based on the interchange of a single base when cation exchange with a phosphate hydrogen is considered as a possibility. This is the mass difference caused by the exchange of a sodiated $\mathrm{dC}$ (311.04 Da) with a deprotonated dA (313.06 Da).

\section{Analysis of LC/MS Data from Enzymatic Digests}

Enzymatic digestion produces a series of ODN fragments that are formed by successive cleavage of terminal nucleotides. Individual reaction products are differentiated through the observation of specific ions whose $m / z$ are specific for the position of a particular modified guanine residue. Digest fragments are described using the system of nomenclature proposed by McLuckey and Habibigoudarzi [23]. Briefly, ions generated by 3'-PDE digestion are referred to as B-type ions and those formed by the $5^{\prime}$-PDE are referred to as Y-type ions. The number following the B- or Y-designation specifies the number of individual nucleotides in that particular digest fragment. A Y7 or B7 ion is an ODN composed of seven nucleotides.

A series of single ion chromatograms demonstrating the detection of several of the ODN fragments generated during the $3^{\prime}$-enzymatic digestion of the single modified ODN 5'-CTCGGCG(AF)CCATC-3' (P1) is shown in Figure 3. The B6 fragment gives an $(\mathrm{M}-$ $2 \mathrm{H})^{2-}$ ion at $m / z 882.7$ that corresponds to a $5^{\prime}-$ CTCGGC-3' formed by the loss of the modified guanine. The observation of this ODN fragment containing the two unmodified guanines confirms the identity of this particular single modified positional isomer. Very little separation of the ODN fragments is achieved in this step. The primary purpose of the LC separation is to isolate the ODN fragments from the other components of the digest mixture that suppress ionization and compromise sensitivity. The retention times of ODN fragments formed in the digestion of di-AF modified dodecamers show longer retention times (up to $7 \mathrm{~min}$ ).

LC/MS spectra of the $5^{\prime}$ - and $3^{\prime}$-digests of the P1 ODN are shown in Figure $4 a$ and $b$. The $Y 8$ and $Y 7$ ODN digest fragments in Figure 4a are formed by the cleavage of one and two unmodified guanine nucleotides from the 5 -terminus, respectively. Both the $\mathrm{Y} 8$ and Y7 ODN fragments form $(\mathrm{M}-2 \mathrm{H})^{2-}$ ions at $m / z$ 1273.6 and 1109.1, respectively. These fragments give $(\mathrm{M}-3 \mathrm{H})^{3-}$ ions at $\mathrm{m} / \mathrm{z} 848.7$ and 739.1 , respectively, as well. The sequence/composition of the $\mathrm{Y} 7$ suggested by the $m / z$ of the $(\mathrm{M}-2 \mathrm{H})^{2-}$ and $(\mathrm{M}-3 \mathrm{H})^{3-}$ ions correspond to $5^{\prime}-\mathrm{CG}(\mathrm{AF}) \mathrm{CCATC}-3^{\prime}$, verifying that the $\mathrm{G}$ closest to the $3^{\prime}$ terminus is modified by the aminofluorene. Figure $4 \mathrm{~b}$ is an ESI-TOF mass spectrum of digest fragments of the $3^{\prime}$-exonuclease digestion of the P1 ODN 5'-CTCGGCG(AF)CCATC-3' after 40 min di- 


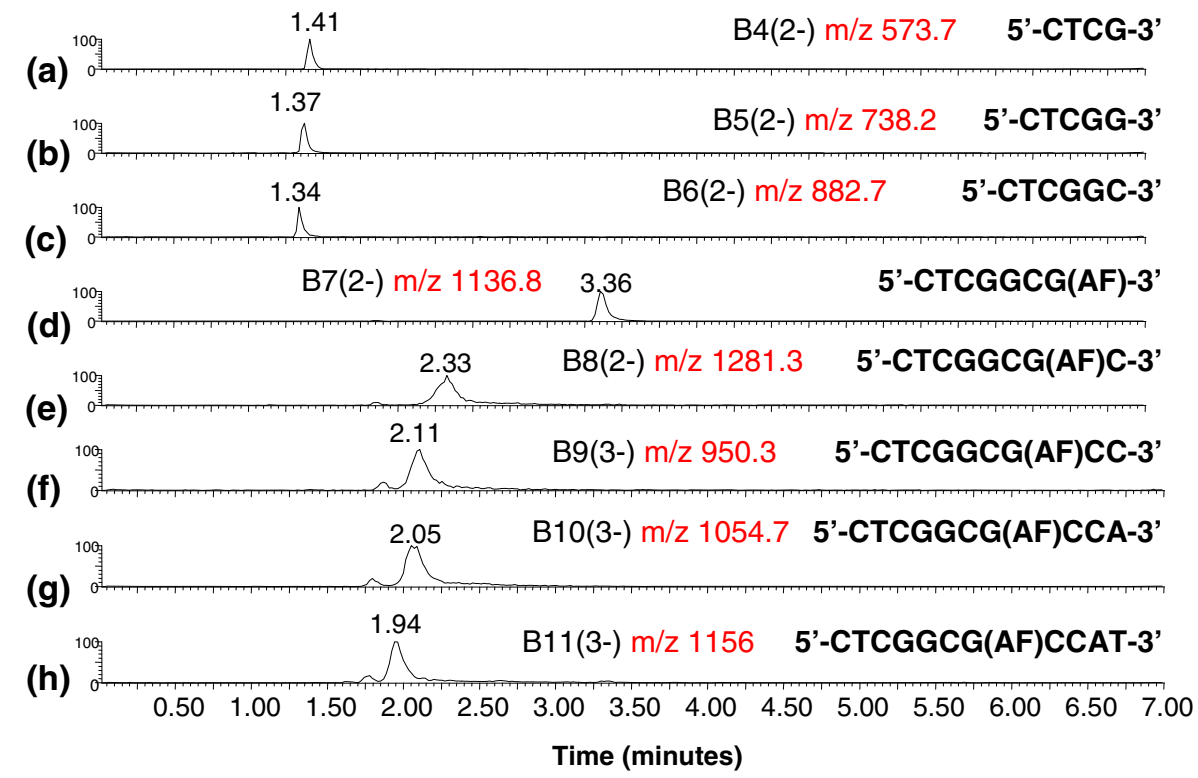

Figure 3. LC/MS single ion chromatograms of the predominant ions formed by ODN digest fragments generated during the 3 "-exonuclease digestion of product 1 . Chromatograms (a)-(e) were acquired after $40 \mathrm{~min}$ digestion time, and (f)-(h) after $20 \mathrm{~min}$.

gestion time. The Figure $4 \mathrm{~b}$ mass spectrum indicates that the digestion of the dodeca ODN is nearly complete after $40 \mathrm{~min}$. The ESI-TOF mass spectrum of the singlymodified ODN (at G3, P1) shows a B6 digest fragment corresponding to the cleavage of the AF-modified guanine at $\mathrm{m} / \mathrm{z} 882.7$ discussed above. Thus, the LC/MS analyses of both $3^{\prime}$ - and $5^{\prime}$-digests are consistent in specifying that the $G$ closest to the $3^{\prime}$ terminus is modified. The positions of the AF modification in the singly-modified ODNs P2 and P3 were established in a similar manner.

The 5'-PDE and 3'-PDE behaved differently when a modified guanine was encountered in the digestion of a dodeca ODN modified by a single AF. The rate of the

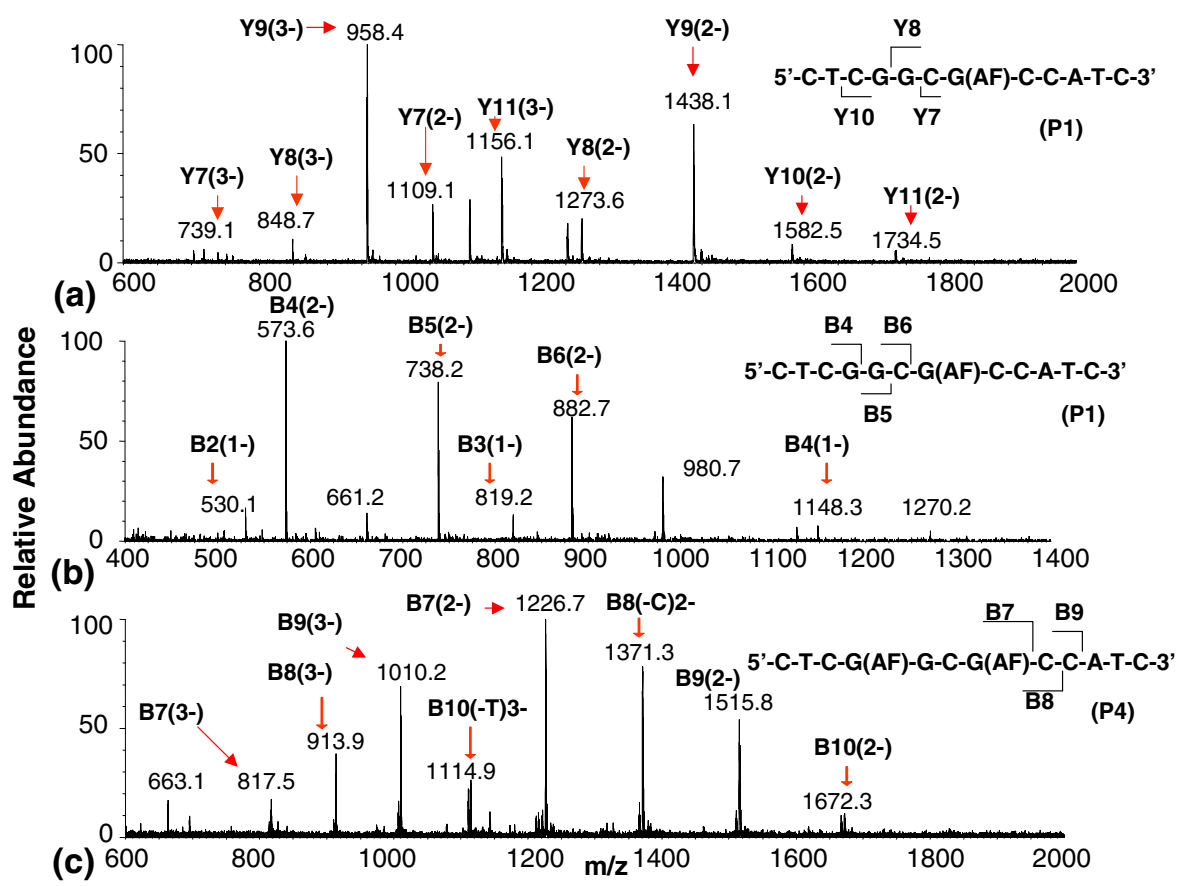

Figure 4. ESI-TOF mass spectrum of the hydrolysis fragments formed from (a) the $5^{\prime}$ - exonuclease digestion of product 1 acquired after $30 \mathrm{~min}$; (b) the hydrolysis fragments formed from the 3 '-exonuclease digestion of product 1 after $40 \mathrm{~min}$; (c) the hydrolysis fragments formed from the $3^{\prime}$-exonuclease digestion of product 4 after $40 \mathrm{~min}$. 
5 '-PDE digestion was found to be slowed greatly when a modification was encountered, and only small abundances of ions formed by the loss of a modified guanine nucleotide were observed. It was found throughout the course of this study that the 3'-PDE would cleave AF-modified nucleotides in dodeca ODNs with a single site of modification, but not those with two modifications. This is demonstrated in the analysis of 5'-CTCG(AF)GCG(AF)CCATC-3' described below.

Figure $4 \mathrm{c}$ is an ESI-TOF mass spectrum of $3^{\prime}-\mathrm{PDE}$ digest spectrum of the ODN, $5^{\prime}$-CTCG(AF)GCG(AF) CCATC-3' (P4), which contains two AF groups. The sequence of this ODN is identical to that of the P1 ODN 5'-CTCGGCG(AF)CCATC-3', whose analysis is described above except for the second AF modification on the guanine (G1) nearest the 5'-terminus. The 3'-PDE digest of this di-AF modified ODN was analyzed after 40 min reaction time, consistent with that of the singlymodified ODN described above. The digestion appears to stop once the enzyme encounters the first modification from the $3^{\prime}$-end of the chain in contrast to the digestion of 5'-CTCGGCG(AF)CCATC-3', where the enzyme is observed to cleave this modified nucleotide. The shortest ODN fragment observed in Figure $4 \mathrm{c}$ is the B7 fragment that gives $(\mathrm{M}-2 \mathrm{H})^{2-}$ and $(\mathrm{M}-3 \mathrm{H})^{3-}$ ions at $\mathrm{m} / \mathrm{z} 1226.7$ and 817.5, respectively. Ions formed from the B6 fragment are not observed here or at digestion times up to $2 \mathrm{~h}$. The $3^{\prime}$-PDE digestion of all three di-AF modified ODNs used in this study were slowed considerably when the first modified nucleotide was encountered. Most ODN fragments that are observed after the formation of the B7 ODN can be attributed to endonuclease activity and do not provide any sequence information.

\section{Competition between Exo- and Endonuclease Activity}

The amount of sequence data one obtains in the analysis of an enzymatic digestion is limited by the onset of endonuclease activity of the exonuclease preparation being used. Endonucleases are enzymes that cleave phosphodiester bonds randomly throughout an ODN. Earlier ODN digest analyses carried out by MALDI suggest that $3^{\prime}$ - and $5^{\prime}$-exonucleases themselves exhibit endonuclease activity, or that the exonuclease preparations are contaminated with proteins that promote endonuclease activity [10-12] and thus limit the amount of sequence information that may be obtained from an ODN analysis. We identified two factors in this study that appear to enhance exonuclease activity relative to endonuclease activity. One was the lower temperature of the digestion, that being $24^{\circ} \mathrm{C}$ relative to $37^{\circ} \mathrm{C}$ as described in other investigations $[9,10,13]$. The second factor was a lower enzyme to substrate ratio in the digestion mixture. In these experiments, we used an enzyme substrate ratio of $1.9 \times 10^{-5}$ units of enzyme per picomole of ODN, whereas in other studies enzyme:
ODN ratios as high as $7.5 \times 10^{-4}$ units of snake venom phosphodiesterase enzyme per picomole of substrate have been used to carry out ODN digestions [13]. When these conditions were implemented for the 5'-PDE digestions, only very small abundances of ODNs formed by the loss of a modified guanine nucleotide are observed.

We have digested more than 50 dodeca ODNs with different sequences containing a single AF modification [4,@1,@4].@n@ach@ase@vidence@or@he@leavage@f@he modified guanine was observed when ODNs with one AF group were subjected to 3 '-exonuclease digestion. These results and those of other investigators described above suggest that the nature of the modification/DNA damage is more important factor in determining the ability to digest modified ODNs than the sequence of bases surrounding the modification. Further, our findings are inconsistent with all but one ladder sequencing study that indicate sequencing past a damaged ODN with a $3^{\prime}$-PDE is not possible before the onset of endonuclease activity. The exception was a study conducted by Pieles et al. who observed the cleavage of a 2'-O-methyladenosine by a 3 '-PDE [9]. Studies of ODNs modified by 2 -amino1-methyl-6-phenylimidazo[4,5-b]pyridine (PhIP), 8oxoguanine, and depurinated DNA strands by MALDI have shown no evidence for cleavage of damage sites by a 3'-PDE [10-13]. Studies of ODNs containing consecutive 8-oxoguanine and deletion sites where bases have been replaced with formyl groups illustrated that digestion ceased when the cluster damage was encountered. The 5'-PDE digestion ceased one nucleotide before the 8-oxoguanine damage site in these ODNs with cluster damage [11].

\section{Differentiation of di-AF Modified ODN Positional Isomers}

Despite the inability to cleave modified nucleotides in dodeca ODNs with two modifications using the 3'-PDE, we were able to observe sequence-specific fragments in these digest analyses that would permit the sequence assignment of all three di-AF modified ODN reaction products. All three di-modified ODNs studied here could be digested by both exonucleases to the point where the modification was exposed at the terminus within $40 \mathrm{~min}$ digestion time. Two of the three dimodified products could be confirmed as 5'-CTCGG (AF)CG(AF)- CCATC-3' (P5) and 5'-CTCG(AF)G(AF) CGCCATC-3' (P6) by LC/MS due to the observation of ODN fragments formed by the loss of the unmodified guanine. The 3'-PDE digest of P6 showed an ion at $\mathrm{m} / \mathrm{z}$ 917.2 after $40 \mathrm{~min}$ digestion time that corresponds to the doubly-charged B5 ODN fragment 5 '-CTCG(AF)G(AF)$3^{\prime}$. The 3'-PDE digest of $\mathrm{P} 4$ produced ions at $\mathrm{m} / \mathrm{z} 817.5$ and 1226.7 whose masses are consistent with the $(\mathrm{M}-$ $3 \mathrm{H})^{3-}$ and $(\mathrm{M}-2 \mathrm{H})^{2-}$ generated from the B7 ODN fragment $5^{\prime}$-CTCG(AF)GCG(AF)-3' (Figure 4c). LCTOF-MS analysis of the $5^{\prime}$-PDE digest show significant 


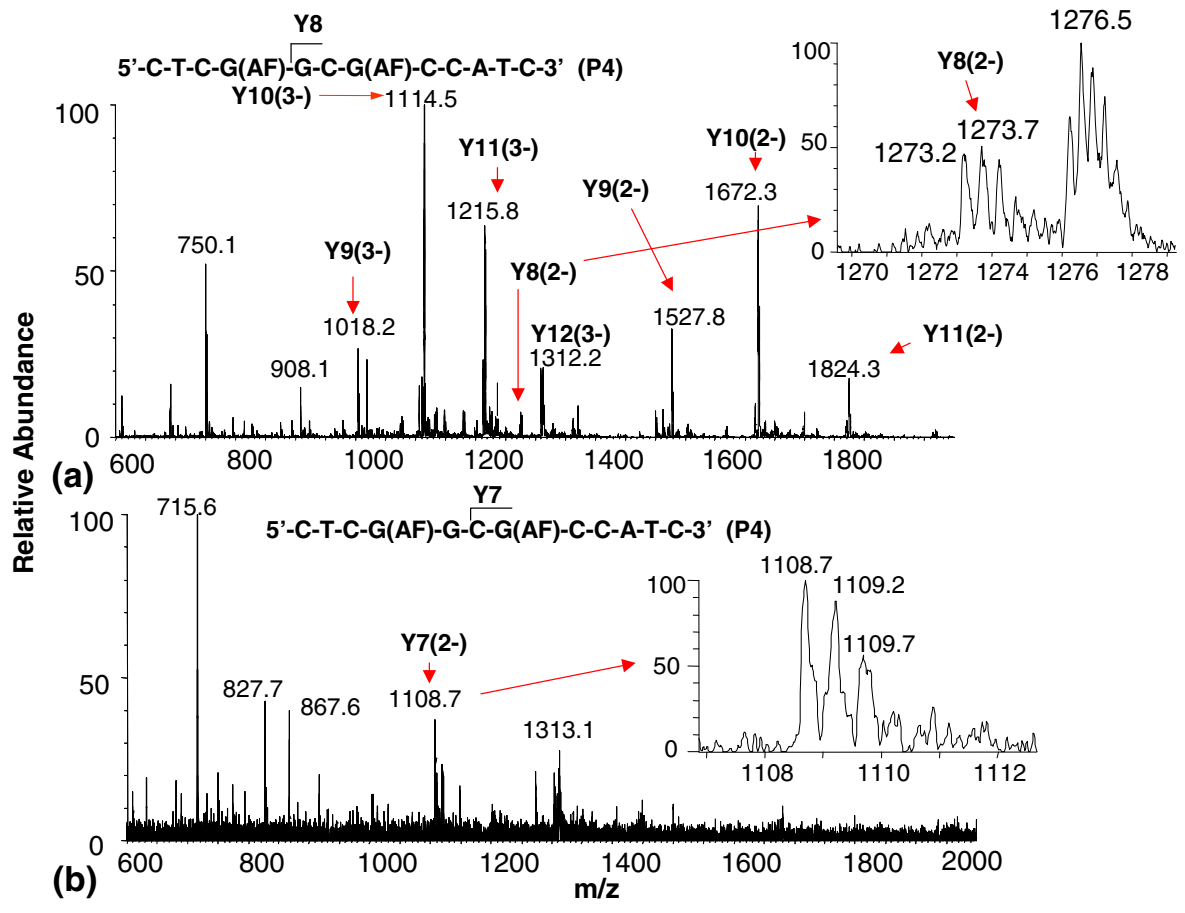

Figure 5. ESI-TOF mass spectrum of (a) the hydrolysis fragments formed from the 5'-exonuclease digestion of product 4 after $80 \mathrm{~min}$. The inset shows the $\mathrm{Y} 8$ fragment formed by the loss of a modified guanine nucleotide (spectrum summed over 3.5 to $7 \mathrm{~min}$ retention time); (b) of the hydrolysis fragments formed from the $5^{\prime}$-exonuclease digestion of product 4 after $80 \mathrm{~min}$. The inset shows the Y7 fragment formed by the consecutive losses of a modified guanine (G1) and unmodified guanine (G2) nucleotide (spectrum summed over 2.5 to 3.5 min retention time).

abundances of ions at $\mathrm{m} / \mathrm{z} 1018.2$ and 1527.8 whose masses are consistent with the $(\mathrm{M}-3 \mathrm{H})^{3-}$ and $(\mathrm{M}-$ $2 \mathrm{H})^{2-}$ formed from the Y9 ODN fragment $5^{\prime}-\mathrm{G}(\mathrm{AF}) \mathrm{GCG}$ (AF)CCATC-3' (Figure 5a). The data obtained from the LC/MS analyses of the P4 digests and the other two di-AF modified reaction products indicate that the sequence of $\mathrm{P} 4$ is $5^{\prime}$-CTCG(AF)GCG(AF)CCATC-3'. The $5^{\prime}$-PDE digest of this ODN also gave detectable amounts of an ion at $\mathrm{m} / \mathrm{z} 1273.2$ that is consistent with an $(\mathrm{M}-2 \mathrm{H})^{2-}$ ion derived from $5^{\prime}$-GCG(AF)CCATC$3^{\prime}$, Y8, an ODN fragment formed by the loss of a AF-modified guanine nucleotide. The significance of the formation of this $\mathrm{Y} 8$ fragment is discussed in more detail below.

\section{Effect of AF Modification Position on 5'-Exonuclease Digestion Efficiency}

The observation of an ODN fragment formed by the cleavage of an AF-modified guanine nucleotide from $\mathrm{P} 4$ encouraged us to analyze these di-AF modified ODNs after extended digestion times to determine how much sequence information we might be able to obtain. The cleavage of a modified guanine in a longer sequence template to be used for site-specific mutagenesis may be necessary for sequence verification in ODNs containing more than two modifications. No evidence for endonuclease activity was observed for digestion periods up to $120 \mathrm{~min}$. For reaction times up to $120 \mathrm{~min}$, no cleavage of an AF-modified nucleotide from a di-AF modified dodeca ODN was observed using the $3^{\prime}$-PDE. The $5^{\prime}$-PDE digest of the di-modified ODN containing consecutive aminofluorene modifications (P6) did not yield any ions formed by the loss of a modified nucleotide beyond $120 \mathrm{~min}$ of digestion time. However, when the ODNs containing one and two unmodified nucleotides between the modified Gs were analyzed, small abundances of ions formed from ODNs undergoing cleavage of modified nucleotides were observed. ESI-TOF mass spectrum of 5'-CTCGG(AF)CG(AF)CCATC-3' (P5), which is dominated by doubly- and triply-charged ions formed from the Y8 fragment formed by the loss of the unmodified guanine, G1, nearest the $5^{\prime}$-terminus, at $\mathrm{m} / \mathrm{z}$ 1363.2 and 908.5, respectively (Figure 6). However, an $(\mathrm{M}-2 \mathrm{H})^{2-}$ ion derived from the Y6 ODN fragment is observed as well at $\mathrm{m} / \mathrm{z}$ 964.2, which is consistent with the sequence $5^{\prime}-\mathrm{G}(\mathrm{AF}) \mathrm{CCATC}-3^{\prime}$. The observation of this ion suggests that we may be able to cleave modified bases using the $5^{\prime}$-exonuclease in route to verifying the sequence of a specific multiply-modified ODN that is to be used as a template for site-specific mutagenesis studies. The mass analysis of the $5^{\prime}$-PDE digest of 5'-CTCG(AF)GCG(AF)CCATTC-3' (P4) carried out after $80 \mathrm{~min}$ of digestion time indicates that the two unmodified bases between the aminofluorenes may be cleaved in addition to the modified G1 closest to the 5 'terminus. The Figure 5a ESI-TOF mass spectrum shows a small abundance of ions around $\mathrm{m} / \mathrm{z} 1273.7$ 


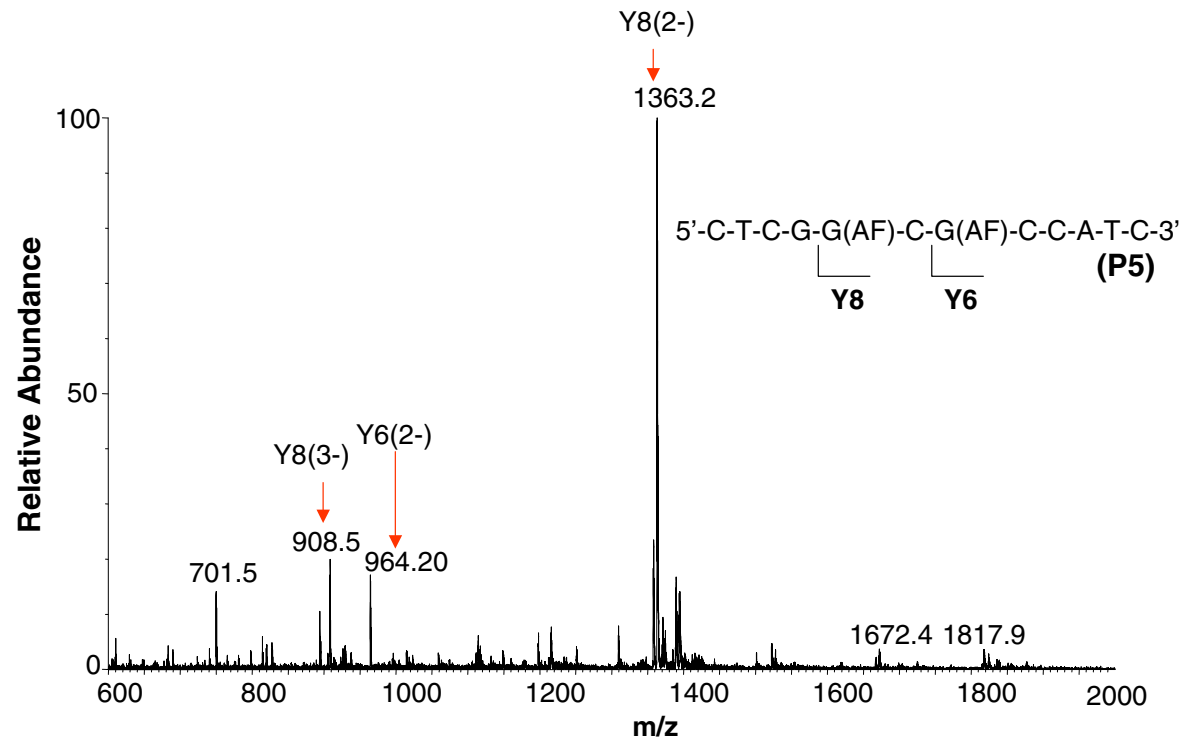

Figure 6. ESI-TOF mass spectrum of the hydrolysis fragments formed from the 5 '-exonuclease digestion of product 5 after $40 \mathrm{~min}$.

whose composition is consistent with the $(\mathrm{M}-2 \mathrm{H})^{2-}$ ion derived from the Y8 ODN fragment $5^{\prime}-\mathrm{GCG}(\mathrm{AF})$ CCATC-3' formed the by the cleavage of the AFmodified $\mathrm{G}$ closest to the $5^{\prime}$-terminus. The observation of a $Y 7$ ion at $m / z 1108.7$ in the Figure $5 b$ ESI-TOF mass spectrum provides confirmation that $\mathrm{G} 2$ is unmodified. The composition of this Y7 (5'-CG(AF)CCATC-3') fragment suggests that it is formed by the loss of an unmodified guanine nucleotide from the $\mathrm{Y} 8$ fragment. This data suggests that when two or more unmodified bases exist between two arylamine modifications in an ODN with multiple modifications, a 5'-PDE may be used to confirm the sequence (location of unmodified guanines surrounded by modifications on the $3^{\prime}$ - and $5^{\prime}$-side) of the reaction product that may be used as a template in site-specific mutagenesis studies.

\section{Conclusions}

An on-line LC/MS method for the sequence analysis of oligonucleotides modified by multiple arylamines has been demonstrated. The method was tested by analyzing six different reaction products formed by the reaction of $\mathrm{N}$-acetoxy- $\mathrm{N}$-(trifluoroacetyl)-2-aminofluorene with 5'-CTCGGCGCCATC-3'. The digestion conditions used in these experiments permitted the observation of ODN fragments formed by the cleavage of damaged bases, unlike earlier mass spectrometry studies of ODN digests. The 3'-PDE digestion (snake venom phosphodiesterase) was found to cleave AF-modified guanines in dodeca ODNs containing a single modification, but not in ODNs with two modifications. The analyses of 5 '-PDE digests of dodeca ODNs modified by two arylamines suggest that modified nucleotides in reaction products with at least one unmodified base between two modifications may be cleaved in route to sequence verification.

\section{References}

1. Shibutani, S.; Suzuki, N.; Tan, X.; Johnson, F.; Grollman, A. P. Influence of Flanking Sequence Context on the Mutagenicity of AcetylaminofluoreneDerived DNA adducts in Mammalian Cells. Biochemistry 2001, 40, 37173722.

2. Singer, B.; Hang, B. Nucleic Acid Sequence and Repair: Role of Adduct, Neighbor Bases and Enzyme Specificity. Carcinogenesis 2000, 21, 10711078 .

3. Zou, Y.; Shell, S. M.; Utzat, C. D.; Luo, C.; Yang, Z.; Geacintov, N. E.; Basu, A. K. Effects of DNA Adduct Structure and Sequence Context on Strand Opening of Repair Intermediates and Incision by UvrABC Nuclease. Biochemistry 2003, 42, 12654-12661.

4. Meneni, S. R.; Shell, S. M.; Gao, L.; Jurecka, P.; Wang, L.; Sponer, J.; Zou, Y.; Chiarelli, M. P.; Cho, B. P. Spectroscopic and Theoretical Insights into Sequence Effects of Aminofluorene-Induced Conformational Heterogeneity and Nucleotide Excision Repair. Biochemistry 2007, 46, 11263-11278.

5. Beland, F. A.; Kadlubar, F. F. Metabolic Activation and DNA Adducts of Aromatic Amines and Nitroaromatic Hydrocarbons. In Handbook of Experimental Pharmacology, Cooper, C. S.; Grover, P. L., Eds.; SpringerVerlag: Heidelberg 1990, pp. 267-325.

6. Stemmler, E. A.; Buchanan, M. V.; Hurst, G. B.; Hettich, R. L. Analysis of Modified Oligonucleotides by Matrix-Assisted Laser Desorption/ Ionization Fourier Transform Mass Spectrometry. Anal. Chem. 1995, 67, 2924-2930.

7. Glover, R. P.; Lamb, J. H.; Farmer, P. B. Tandem Mass Spectrometry Studies of a Carcinogen Modified Oligodeoxynucleotide. Rapid Commun. Mass Spectrom. 1998, 12, 368-372.

8. Chen, H.; Zhang, W.; Song, R.; Ma, H.; Dong, Y.; Sheng, G.; Zhou, Z.; Fu, J. Analysis of DNA Methylation by Tandem Ion-Pair Reversed-Phase High-Performance Liquid Chromatography/Electrospray Ionization Mass Spectrometry. Rapid Commun. Mass Spectrom. 2004, 18, 2773-2778.

9. Pieles, U.; Zurcher, W.; Schar M.; Moser, H. E. Matrix-Assisted Laser Desorption Ionization Time-of-Flight Mass Spectrometry: A Powerful Tool for the Mass and Sequence Analysis of Natural and Modified Oligonucleotides. Nucleic Acids Res. 1993, 21, 3191-3196.

10. Brown, K.; Harvey, C. A.; Turteltaub, K. W.; Shields, S. J. Structural Characterization of Carcinogen-Modified Oligodeoxynucleotide Adducts Using Matrix-Assisted Laser Desorption/Ionization Mass Spectrometry. J. Mass Spectrom. 2003, 38, 68-79.

11. Bourdat, A. G.; Gasparutto, D.; Cadet, J. Synthesis and Enzymatic Processing of Oligodeoxynucleotides Containing Tandem Base Damage. Nucleic Acids Res. 1999, 27, 1015-24.

12. Zhang, L.-K.; Gross, M. L. Matrix-Assisted Laser Desorption/Ionization Mass Spectrometry Methods for Oligodeoxynucleotides: Improvements in Matrix, Detection Limits, Quantification, and Sequencing. J. Am. Soc. Mass Spectrom. 2000, 11, 854-865.

13. Zhang, L.-K.; Ren, Y.; Rempel, D.; Taylor, J. S.; Gross, M. L. Determination of Photomodified Oligodeoxynucleotides by Exonuclease Digestion, Matrix-assisted Laser Desorption/Ionization and Post-Source Decay Mass Spectrometry. J. Am. Soc. Mass Spectrom. 2001, 12, 1127-1135.

14. Null, A. P.; Benson, L. M.; Muddiman, D. C. Enzymatic Strategies for the Characterization of Nucleic acids by Electrospray Ionization Mass Spectrometry. Rapid Commun. Mass Spectrom. 2003, 17, 2699-2706. 
15. Premstaller, A.; Huber, C. G. Factors Determining the Performance of Triple Quadrupole, Quadrupole Ion Trap and Sector Field Mass Spectrometer in Electrospray Ionization Mass Spectrometry. II. Suitability for de Novo Sequencing. Rapid Commun. Mass Spectrom. 2001, 15, 1053-1060.

16. Glover, R. P.; Sweetman, G. M.; Farmer P. B.; Roberts, G. C. Sequencing of Oligonucleotides Using High Performance Liquid Chromatography and Electrospray Mass Spectrometry. Rapid Commun. Mass Spectrom. 1995, 9, 897-901.

17. Wu, H.; Aboleneen, H. Sequencing Oligonucleotides with Blocked Termini Using Exonuclease Digestion and Electrospray Mass Spectrometry. Anal. Biochem. 2000, 287, 126-135.

18. Huber C. G.; Oberacher, H. Analysis of Nucleic Acids by On-line Liquid Chromatography-Mass Spectrometry. Mass Spectrom. Rev. 2001, 20, $310-43$.

19. Burnouf, D.; Koehl, P.; Fuchs, R. P. P. Single Adduct Mutagenesis: Strong Effect of the Position of a Single Acetylaminofluorene Adduct within a Mutation Hot Spot. Proc. Natl. Acad. Sci. U.S.A. 1989, 86, 4147-4151.

20. Melchior, W. B. Jr.; Marques, M. M.; Beland, F. A. Mutations Induced by Aromatic Amine DNA Adducts in pBR322. Carcinogenesis 1994, 15, $889-899$.

21. Koehl, P.; Valladier, P.; Le Fevre, J.-F.; Fuchs, R. P. P. Strong Structural Effect of the Position of a Single Acetylaminofluorene Adduct Within a Mutation Hot Spot. Nucleic Acids Res. 1989, 17, 9531-9541.

22. Jain, N.; Li, Y.; Zhang, Li.; Meneni, S.; Cho, B. P. Probing the Sequence Effects on NarI-induced -2 Frameshift Mutagenesis by Dynamic ${ }^{19} \mathrm{~F}$ NMR, UV, and CD spectroscopy. Biochemistry 2007, 46, 13310-13321.

23. McLuckey, S. A.; Habibigoudarzi, S. Decompositions of MultiplyCharged Oligonucleotide Anions. J. Am. Chem. Soc. 1993, 115, 1208512095.

24. Meneni, S. R.; D’Mello, R. N.; Baker, G.; Gao, L.; Chiarelli, M. P.; Cho, B. P. Sequence Effects of Aminofluorene-Modified DNA Duplexes: Thermodynamic and Circular Dichroism Properties. Nucleic Acids Res. 2006, 34, 755-763. 